\title{
Simultaneous Determination of Five Bile Acids as Potential Biomarkers for Alzheimer's Disease in Mouse Brain and Plasma
}

\author{
Shin KoIKe, Yusuke MiyaJI, Hinako Sano, Natsuki AiKawa, Masayuki KaI, Sakura KaSaHara, \\ Toshihiro SuzukI, Shoichi Nishimoto-Kusunose, and Yuki OgaSAWARA
}

Department of Analytical Biochemistry, Meiji Pharmaceutical University, 2-522-1 Noshio, Kiyose, Tokyo 204-8588, Japan

\begin{abstract}
In this study, we developed an analytical method using LC-MS/MS for the simultaneous determination of five bile acids (BAs) that have been recently reported as candidate diagnostic biomarkers for Alzheimer's disease (AD) or AD related factors in the brain. The measurement of BAs in the brains of healthy mice led to the determination of candidate diagnostic markers for AD, such as cholic acid and deoxycholic acid, and other bile acids, such as chenodeoxycholic acid noted for the ameliorating effect on the symptoms of AD. Significant positive correlations were observed between the brain and plasma concentrations of four BAs in healthy young mice. These results indicate that the BA level in the brain may be estimated by the corresponding BA level in the plasma. Thus, our study suggested that the proposed method for the analysis of the five bile acids would aid in the diagnosis of $\mathrm{AD}$ or in studies that use AD model mice.
\end{abstract}

Keywords Alzheimer's disease, bile acid, biomarker, LC-MS/MS, mouse brain, simultaneous determination

(Received December 4, 2020; Accepted January 19, 2021; Advance Publication Released Online by J-STAGE January 29, 2021)

\section{Introduction}

Bile acids (BAs), the major components of bile, are produced from cholesterol in the hepatocytes of the liver. BAs play fundamental roles in many physiological processes. They are popularly termed as "nutritional detergents" that dissolve lipids and lipid-soluble vitamins. However, recent studies suggest that BAs may also act as signaling molecules, ${ }^{1-3}$ a notion that is in line with the widespread distribution of BA receptors throughout various organisms. BAs and their associated receptors have been detected in human and rodent brains. ${ }^{4}$ Additionally, it has been well studied that BA transporters are crucial for maintaining BA circulation and homeostasis ${ }^{5}$ and specific transporters carefully regulate the entry and removal from the central nervous system. ${ }^{6}$ However, the quantitative and qualitative analyses of BAs in the brain have yet to be explored in depth. Although Zheng et al. identified 20 BAs in rat brain tissue samples, including nine unconjugated BAs and eleven conjugated BAs, only three of the unconjugated BAs have so far been quantified in rat brain. ${ }^{8,9}$ There are also significant discrepancies between the previously reported studies regarding the identification and quantification of BAs in the human brain. ${ }^{10,11}$

Some reports have demonstrated significant changes in the level of free BAs in the plasma of AD patients, such as decreased cholic acid (CA) concentration, ${ }^{11,12}$ increased deoxycholic acid (DCA) concentration, ${ }^{12,13}$ and increased lithocholic acid (LCA) concentration. ${ }^{14}$ These results indicate that these BAs may be useful diagnostic markers of AD. In a recent study carried out on post-mortem brains, Pan et al. observed a significant decrease

$\dagger$ To whom correspondence should be addressed.

E-mail: yo@my-pharm.ac.jp in the taurocholic acid (TCA) concentration in the brains of AD patients, along with significant reductions in the levels of CA and TCA in the brains of AD model mice. ${ }^{11}$ Furthermore, Bazzari et al. indicated that the administration of chenodeoxycholic acid (CDCA) ameliorates neurotoxicity and cognitive deterioration in AD model rats. ${ }^{15}$ However, very few reports have examined the levels of BAs in a healthy brain as well as its homeostasis. The link between the pathology of AD and the concentration of BAs in the blood or brain is yet to be established.

In this study, we focused on the five types of BAs that have previously been reported as potential diagnostic biomarkers for Alzheimer's disease, or have an impact on the symptoms of AD in the brain. We developed a technique for the simultaneous analysis of these five types of BAs by modifying a previously reported LC-MS/MS-based analytical method. We tested the utility of this analytical method in the plasma and brain of healthy young mice.

\section{Experimental}

Chemicals

CA, CDCA, DCA, TCA, and LCA were purchased from Tokyo Kasei (Tokyo, Japan). CA-d4, CDCA-d4, DCA-d4, and TCA-d5 were purchased from C/D/N ISOTOPES, INC. (Pointe-

Abbreviations: BA, bile acid; CA, cholic acid; CDCA, chenodeoxycholic acid; DCA, deoxycholic acid; HPLC, highperformance liquid chromatography; LCA, lithocholic acid; LCMS/MS, liquid chromatography tandem mass spectrometry; MRM, multiple reaction monitoring; PBS, phosphate buffered saline; TCA, taurocholic acid. 
Claire, Quebec, Canada). LCA-d4 was obtained from Cambridge Isotope Laboratories, Inc. (Tewksbury, MA, USA) HPLC-grade methanol, acetonitrile, water, ammonium acetate, ammonium formate, ammonium hydroxide, formic acid, and acetic acid were obtained from Fisher Scientific (St Louis, MO, USA). Oasis HLB SPE cartridges were purchased from Waters (Milford, MA, USA).

\section{Animals}

All the animal procedures were performed following the Guidelines for Animal Experimentation of the Japanese Association for Laboratory Animal Science and were approved by the Institutional Animal Use and Care Committee of Meiji Pharmaceutical University. Eight-week-old male C57BL/6 mice were purchased from CREA Japan, Inc. (Tokyo, Japan). The mice were kept in a $12 \mathrm{~h}$ light/dark cycle with access to food and water ad libitum.

\section{Plasma preparation}

Mouse ( 8 - 10 weeks old) blood obtained by cardiac puncture was collected in pre-chilled tubes containing EDTA, centrifuged at $3000 \mathrm{~g}$ for $10 \mathrm{~min}\left(4^{\circ} \mathrm{C}\right)$ and the resultant plasma was transferred to screw-capped vials prior to storage at $-20^{\circ} \mathrm{C}$ for analysis.

\section{Extraction of bile acids from brain tissue}

After blood sampling from the heart of the mice and careful intracardiac perfusion with PBS to remove the blood from the brain capillaries, the whole-brain $(0.4-0.6 \mathrm{~g})$ samples from C57BL/6J mice were immediately removed and collected into individual tubes and stored in a freezer at $-80^{\circ} \mathrm{C}$.

The brain samples were thawed at $4{ }^{\circ} \mathrm{C}$ before the extraction procedure.

Each whole-brain sample was weighed and homogenized in $2 \mathrm{~mL}$ of $5 \mathrm{mM}$ phosphate buffer ( $\mathrm{pH}$ 7.4) using Polytoron (3 times, $30 \mathrm{~s})$ following ultrasonic treatment (Branson, Sonifire) at $4^{\circ} \mathrm{C}$ ( 3 times for $30 \mathrm{~s}$ at $30 \mathrm{~s}$ intervals). The mouse brain suspension was gradually added to $7 \mathrm{~mL}$ of ethanol at $60^{\circ} \mathrm{C}$ with continuous mixing. After centrifugation at $10000 \mathrm{~g}$ for $5 \mathrm{~min}$ at $4^{\circ} \mathrm{C}, 5 \mathrm{~mL}$ of the supernatant was dried under vacuum. The residue was dissolved in $0.5 \mathrm{~mL}$ of an ethanol/ acetonitrile (1:1) mixture.

\section{Pretreatments of the samples}

First, $10 \mu \mathrm{L}$ of a mixture of deuterated standards containing $0.5 \mathrm{ng} / \mu \mathrm{L}$ CDCA-d4, $0.5 \mathrm{ng} / \mu \mathrm{L}$ LCA-d4, $0.5 \mathrm{ng} / \mu \mathrm{L}$ TCA-d5, $0.5 \mathrm{ng} / \mu \mathrm{L}$ DCA-d4, and $0.5 \mathrm{ng} / \mu \mathrm{L}$ CA-d 4 in methanol $/ \mathrm{H}_{2} \mathrm{O}$ (1:1) was added to $0.5 \mathrm{~mL}$ of each calibration standard solution, $0.5 \mathrm{~mL}$ of concentrated mouse brain extracts, and $0.2 \mathrm{~mL}$ of mouse plasma. Subsequently, the spiked solutions were loaded on pre-conditioned solid-phase extraction cartridges (OASIS HLB, $60 \mathrm{mg} / 3 \mathrm{~mL}$ ). Each cartridge was washed with $2 \mathrm{~mL}$ of $5 \%$ methanol and the BAs were eluted with $1 \mathrm{~mL}$ of methanol and $1 \mathrm{~mL}$ of acetonitrile. The collected $2 \mathrm{~mL}$ solution was concentrated using a vacuum evaporator and redissolved in $100 \mu \mathrm{L}$ of methanol/acetonitrile $(1: 1)$ for the brain samples or acetonitrile $/ \mathrm{H}_{2} \mathrm{O}$ (1:1) for the plasma samples and other standards. A $10 \mu \mathrm{L}$ aliquot of each sample was analyzed by LC-MS/MS.

\section{LC-MS/MS system}

The LC-MS system consisted of two LC-30AD pumps, a SIL30AC auto-sampler, a CTO-20A column oven, a CBM-20A system controller, and a triple quadrupole mass spectrometer LCMS-8040 (Shimadzu, Kyoto, Japan). A reversed-phase column (ODS-4, C18, $3.0 \times 150 \mathrm{~mm}$, GL Sciences, Tokyo, Japan) was used for the chromatographic separations. The chromatogram acquisition, detection of mass spectral peaks, and waveform processing were performed using the LabSolutions Insight software (Shimazu, Ver. 2).

\section{Liquid chromatographic conditions}

Five BAs were separated according to a previously reported LC-MS/MS method ${ }^{16}$ with some modifications. The mobile phase consisted of a 2:8-mixture of acetonitrile and $10 \mathrm{mM}$ ammonium formate buffer ( $\mathrm{pH}$ 4.0) (A) and an 8:2-mixture of acetonitrile and $40 \mathrm{mM}$ ammonium formate buffer ( $\mathrm{pH} 4.0)(\mathrm{B})$, at a total flow rate of $0.6 \mathrm{~mL} / \mathrm{min}$. The effects of the mobile phase composition and gradient programs on the chromatography and MS signals were investigated. The five BAs were separated by elution with the following gradient program: $0-3.0 \mathrm{~min}$, $15-27 \%$ B; $3.0-8.0$ min, $27-45 \%$ B; 8.0 - 15.5 min, $45-$ $75 \% \mathrm{~B}$; and $15.5-20.0 \mathrm{~min}, 75 \% \mathrm{~B}$. The column oven was maintained at $40^{\circ} \mathrm{C}$. The injection volume of all the samples was $10 \mu \mathrm{L}$. The peak area of each quantified ion was calculated and normalized to that of a deuterated internal standard [CDCA-d4, DCA-d4, TCA-d5, LCA-d4, and CA-d4, $0.05 \mathrm{ng} / \mu \mathrm{L}$ each, in methanol/ $\left.\mathrm{H}_{2} \mathrm{O}(1: 1)\right]$. Further analyses were performed using normalized values.

\section{Method validation}

Linearity was evaluated for each BA over a concentration range of 0.5 to $1000 \mathrm{nM}$. The limit of detection (LOD) was determined with a signal-to-noise $(S / N)$ ratio $>3$, and the limit of quantification (LOQ) was determined with a $S / N$ ratio $>6$ and $<20 \%$ coefficient of variation (CV, standard deviation divided by the mean). The matrix effect was evaluated by comparing the integrated peak areas of the deuterated standards spiked in the calibration standard with those spiked in the brain and plasma samples.

The precision and accuracy were validated by intra-day (five replicates analyzed on the same day) and inter-day (one replicate per day over five days) variations. The recoveries of the five BAs were determined at $20 \mathrm{ng} / \mathrm{g}$ or $100 \mathrm{nmol} / \mathrm{L}$ concentration each by comparing the responses of BAs from the spiked mouse brain extracts or mouse plasma with those of the corresponding unspiked extracts or plasma, respectively $(n=5)$.

\section{Mass spectrometry conditions}

The LC effluent was analyzed by mass spectrometry using an electrospray ionization (ESI) interface operating in the negative ion mode. The source temperature was $400^{\circ} \mathrm{C}$ and the ion spray voltage was $4.5 \mathrm{kV}$. Nitrogen was used as the nebulizer and drying gas. The tandem mass spectrometer was tuned in the multiple reaction monitoring (MRM) mode to monitor mass transitions in the negative ion mode: CA $\mathrm{m} / \mathrm{z} 453.3 \mathrm{CM}+$

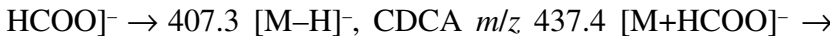
$391.2[\mathrm{M}-\mathrm{H}]^{-}$, DCA $\mathrm{m} / \mathrm{z} 437.4[\mathrm{M}+\mathrm{HCOO}]^{-} \rightarrow 391.2[\mathrm{M}-\mathrm{H}]^{-}$, TCA $\mathrm{m} / \mathrm{z}, 514.3[\mathrm{M}-\mathrm{H}]^{-} \rightarrow 514.3[\mathrm{M}-\mathrm{H}]^{-}, \quad$ LCA $\mathrm{m} / \mathrm{z} \quad 421.5$ $[\mathrm{M}+\mathrm{HCOO}]^{-} \rightarrow 375.5[\mathrm{M}-\mathrm{H}]^{-}$.

\section{Statistical analysis}

Values have been presented as mean \pm standard deviation (SD). Pearson correlation coefficients were used to statistically evaluate the correlation of the BA concentrations between the mouse brain and plasma. A $p$ value of less than 0.05 was considered statistically significant. 


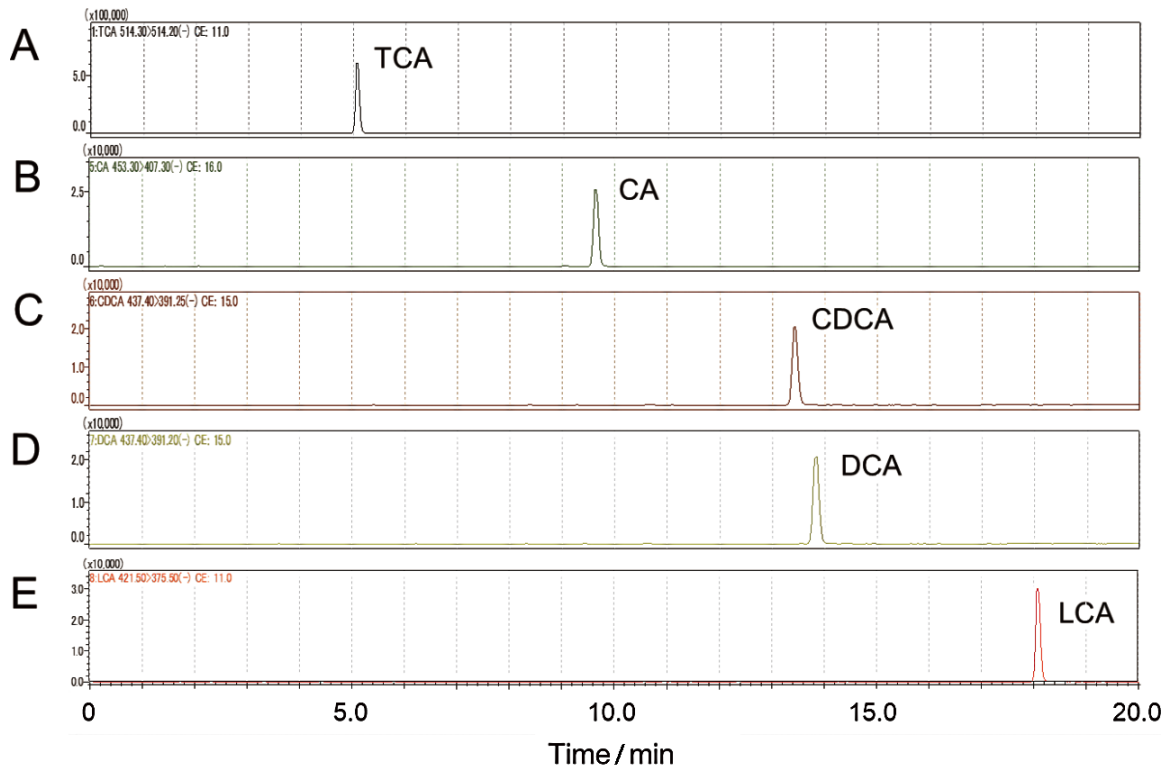

Fig. 1 LC-MS/MS chromatograms of the five standard bile acids. MRM mode (negative): the concentration of each standard bile acid is $500 \mathrm{nM}$. The LC-MS/MS conditions are given in the Experimental section. A: TCA, B: CA, C: CDCA, D: DCA, and E: LCA.

Table 1 Standard curves of five BAs

\begin{tabular}{lrlccc}
\hline $\begin{array}{c}\text { Bile } \\
\text { acid }\end{array}$ & $\begin{array}{c}\text { Retention } \\
\text { time/min }\end{array}$ & Linearity & $\begin{array}{c}\text { Correlation } \\
\text { coefficient, } \\
r^{2}\end{array}$ & $\begin{array}{c}\text { Linear } \\
\text { range/ } \\
\mathrm{nmol} \mathrm{L}^{-1}\end{array}$ \\
\hline TCA & 5.0 & $y=2551123 x-697.67$ & 0.995 & $0.5-1000$ \\
CA & 9.6 & $y=1599276 x+956.18$ & 0.999 & $1.0-1000$ \\
CDCA & 13.4 & $y=955075 x+4753.8$ & 0.999 & $1.0-1000$ \\
DCA & 13.9 & $y=915547 x+273.39$ & 0.999 & $1.0-1000$ \\
LCA & 18.1 & $y=429341 x+535.92$ & 0.995 & $2.5-1000$ \\
\hline
\end{tabular}

a. $y$ indicates the MS/MS signal peak, and $x$ indicates the concentration of the target. The other LC-MS/MS conditions are described in the Experimental section.

\section{Results and Discussion}

Over the last decade, liquid chromatography-tandem mass spectrometry (LC-MS/MS) has gained popularity for the quantification of free BAs and their conjugates in various matrices, such as plasma, blood, urine, and liver. ${ }^{17-20}$ Numerous reports have described different methods for the quantitative determination of BAs in plasma or serum samples obtained during in vivo studies. However, the information on suitable methods for the determination of BAs in brain samples is limited.

We examined the mobile phases and gradient programs to separate CA, TCA, CDCA, DCA, LCA and other BAs based on a previous method, ${ }^{16}$ which aimed to analyze 20 representative BAs using an ultra performance LC-MS/MS system with an exclusive column. As shown in Fig. 1, five BAs could be separated within $20 \mathrm{~min}$ and detected with high sensititvity by a gradient condition with eluents consisting of formate ammonium buffer ( $\mathrm{pH}$ 4.0) and acetonitrile using a conventional LC-MSMS system. We also confirmed that the five BAs could be separated from the other nine bile acids, including tauro- $\beta$ muricholic acid, $\beta$-mucholic acid, tauroursodeoxycholic acid,
Table 2 Validation results for the determination of five BAs

\begin{tabular}{crcccc}
\hline $\begin{array}{c}\text { Bile } \\
\text { acid }\end{array}$ & $\begin{array}{c}\text { Concentration/ } \\
\mathrm{nmol} \mathrm{L}^{-1}\end{array}$ & $\begin{array}{c}\text { Intra-day } \\
\text { RSD, \% }\end{array}$ & $\begin{array}{c}\text { Inter-day } \\
\text { RSD, \% }\end{array}$ & $\begin{array}{c}\text { LOD/ } \\
\mathrm{nmol} \mathrm{L}^{-1}\end{array}$ & $\begin{array}{c}\text { LOQ/ } \\
\mathrm{nmol} \mathrm{L}^{-1}\end{array}$ \\
\hline TCA & 10 & 3.13 & 5.50 & 0.1 & 0.5 \\
& 100 & 1.77 & 4.79 & & \\
& 1000 & 4.32 & 3.33 & & \\
$\mathrm{CA}$ & 10 & 4.10 & 7.94 & 0.2 & 1.0 \\
& 100 & 3.84 & 6.96 & & \\
$\mathrm{CDCA}$ & 1000 & 4.06 & 3.10 & & \\
& 10 & 6.84 & 5.51 & 0.2 & 1.0 \\
& 100 & 3.72 & 3.83 & & \\
DCA & 1000 & 2.53 & 3.62 & & \\
& 10 & 6.49 & 6.69 & 0.2 & 1.0 \\
& 100 & 3.68 & 2.44 & & \\
LCA & 1000 & 2.67 & 2.21 & & \\
& 10 & 6.56 & 6.61 & 1.0 & 2.5 \\
& 100 & 4.92 & 4.17 & & \\
\hline
\end{tabular}

a. Injection volume, $10 \mu \mathrm{L}(n=4)$.

taurochenodeoxycholic acid, taurodeoxycholic acid, glycodeoxycholic acid, glycochenodeoxycholic acid, ursodeoxycholic acid, and hyodeoxycholic acid (Supporting Information, Fig. S1). Free bile acids were clearly detected as $[\mathrm{M}+\mathrm{HCOO}]^{-}$ in the results of the precursor ion scan with ammonium formate ( $\mathrm{pH} 4.0$ ) in the eluent. The highest sensitivities were obtained for all the five target BAs when they were detected as undecomposed $[\mathrm{M}-\mathrm{H}]^{-}$for each product ion under the present method with MRM mode.

As depicted in Table 1, good linear calibration curves for the five BAs were obtained with each concentration range at $\mathrm{nM}$ level (a limit of quantification ranging from 0.5 to $2.5 \mathrm{nmol} / \mathrm{L}$ ), and the calculated coefficient of determination $\left(r^{2}\right)$ being greater than 0.99 under these conditions.

The repeatability and sensitivity of the proposed method were examined using standard solutions of the five BAs (Table 2). 
The intra- and inter-assay precision of the standard solution of the five BAs resulted in relative standard deviations (RSD) of $0.69-6.84$ and $2.21-7.94 \%$, respectively. For a $10 \mu \mathrm{L}$ injection volume of the sample solution, the LOD and LOQ were: $10 \mathrm{fmol}(0.2 \mathrm{nM})$ and $50 \mathrm{fmol}(1.0 \mathrm{nM})$ for CA, CDCA, DCA; $50 \mathrm{fmol}(1.0 \mathrm{nM})$ and $125 \mathrm{fmol}(2.5 \mathrm{nM})$ for LCA; $5 \mathrm{fmol}$ $(0.1 \mathrm{nM})$ and $25 \mathrm{fmol}(0.5 \mathrm{nM})$ for TCA, respectively.

As shown in Table 3, the recoveries for the five BAs $(20 \mathrm{ng} / \mathrm{g}$ added) were $81-98 \%$ in the mouse brain. The intra-day and inter-day RSD of the present method for the five BAs in the brain samples were $2.48-6.21 \%$ and $6.05-9.43 \%$, respectively. In the mouse plasma, the recoveries for the five BAs $(100 \mathrm{nmol} / \mathrm{L}$ added) were $84-101 \%$. The intra-day and inter-day RSD of the present method for the five BAs in the brain samples were $2.42-6.61 \%$ and $4.21-7.72 \%$, respectively.

The amount of BAs in the mouse brain was very small and the concentrations were much lower than those in the plasma. In fact, CA and TCA could only be detected in brain samples

Table 3 Assay precision and recovery for five BAs in mouse brain samples

\begin{tabular}{lccc}
\hline Bile acid & $\begin{array}{c}\text { Intra-day } \\
\text { precision }(\mathrm{RSD}, \%)^{\mathrm{a}}\end{array}$ & $\begin{array}{c}\text { Inter-day } \\
\text { precision }(\mathrm{RSD}, \%)^{\mathrm{a}}\end{array}$ & $\begin{array}{c}\text { Recovery, } \\
\%^{\mathrm{b}}\end{array}$ \\
\hline TCA & 2.48 & 6.05 & $97.85 \pm 7.05$ \\
CA & 5.49 & 9.43 & $98.34 \pm 9.71$ \\
CDCA & 6.21 & 7.67 & $81.20 \pm 4.49$ \\
DCA & 5.63 & 7.55 & $83.99 \pm 6.75$ \\
LCA & LOQ $>$ & LOQ $>$ & $92.26 \pm 8.40$ \\
\hline
\end{tabular}

a. $n=5$.

b. Mean \pm SD. prepared by the extraction and concentration procedures previously used for the BA determination in the rat brain ${ }^{8}$ or mouse liver. ${ }^{16}$ Therefore, we attempted to efficiently extract BAs by completely disintegrating the brain and increasing the concentration ratio of the extracts. As a result, the peak area of LCA was detected below the LOQ; however, the peak areas of the four BAs in mouse brains were detected above the LOQ by

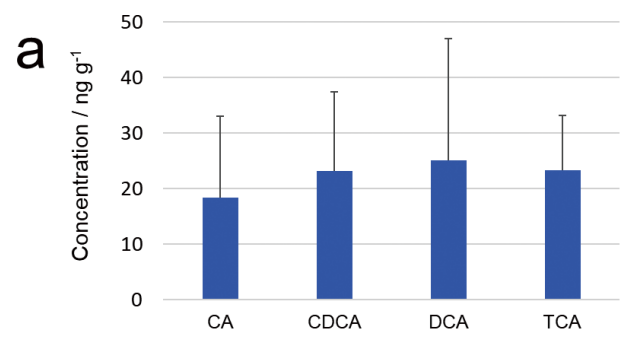

b

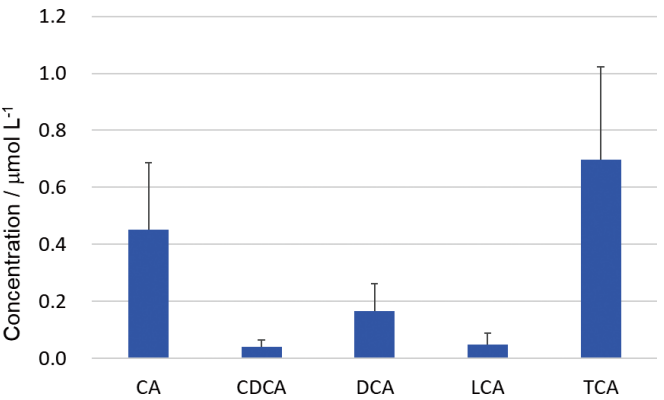

Fig. 3 Bile acid concentrations in mouse brain (a) and plasma (b). The values are presented as mean $\pm \mathrm{SD}(n=15)$. Error bars represent SD.

(a) Brain

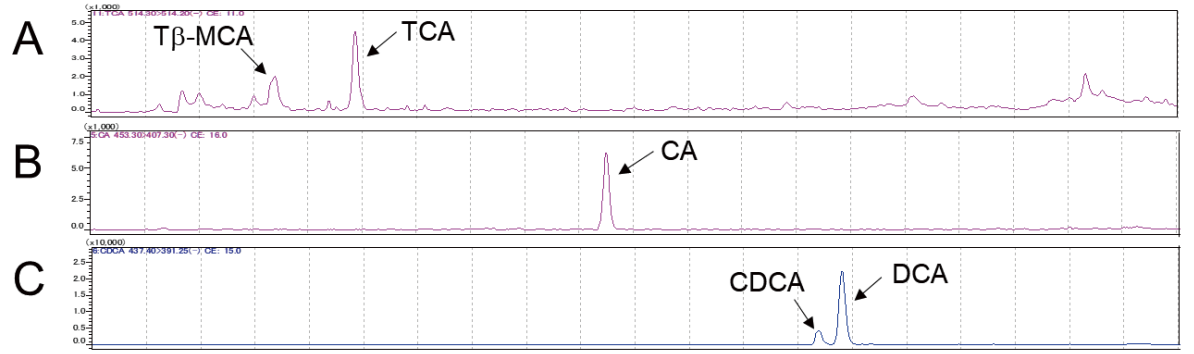

(b) Plasma

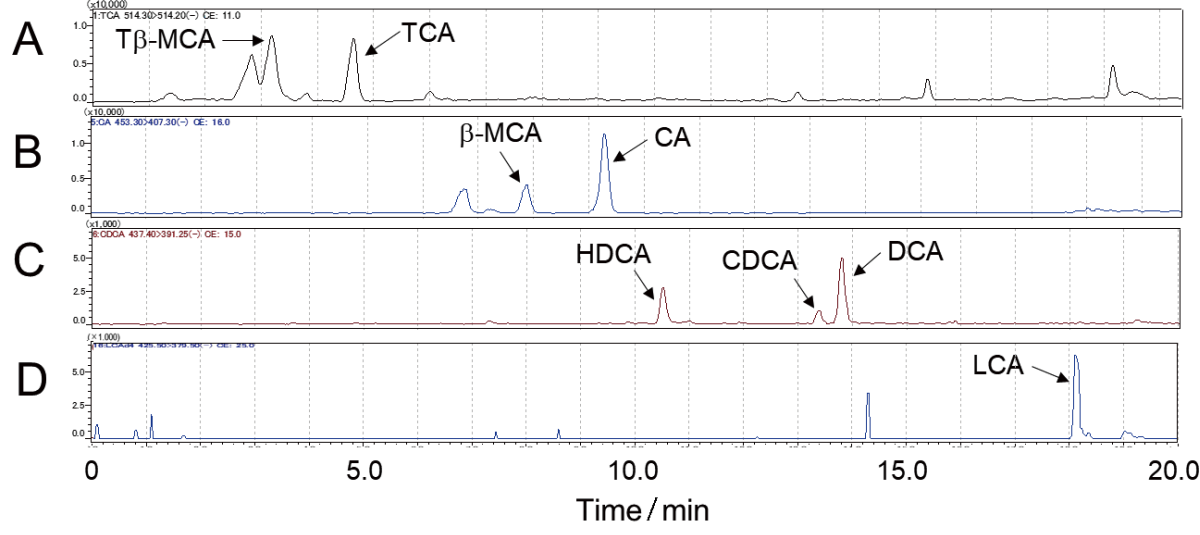

Fig. 2 Typical MRM chromatograms of the mouse brain (a) and plasma (b) samples. The arrows indicate each identified peak. A: $\mathrm{m} / z, 514.3 \rightarrow 514.3$, B: $\mathrm{m} / z, 453.3 \rightarrow 407.3$, C: $\mathrm{m} / z$ 437.4 $\rightarrow$ 391.2, and $\mathrm{D}: m / z 421.5 \rightarrow 375.5$ 

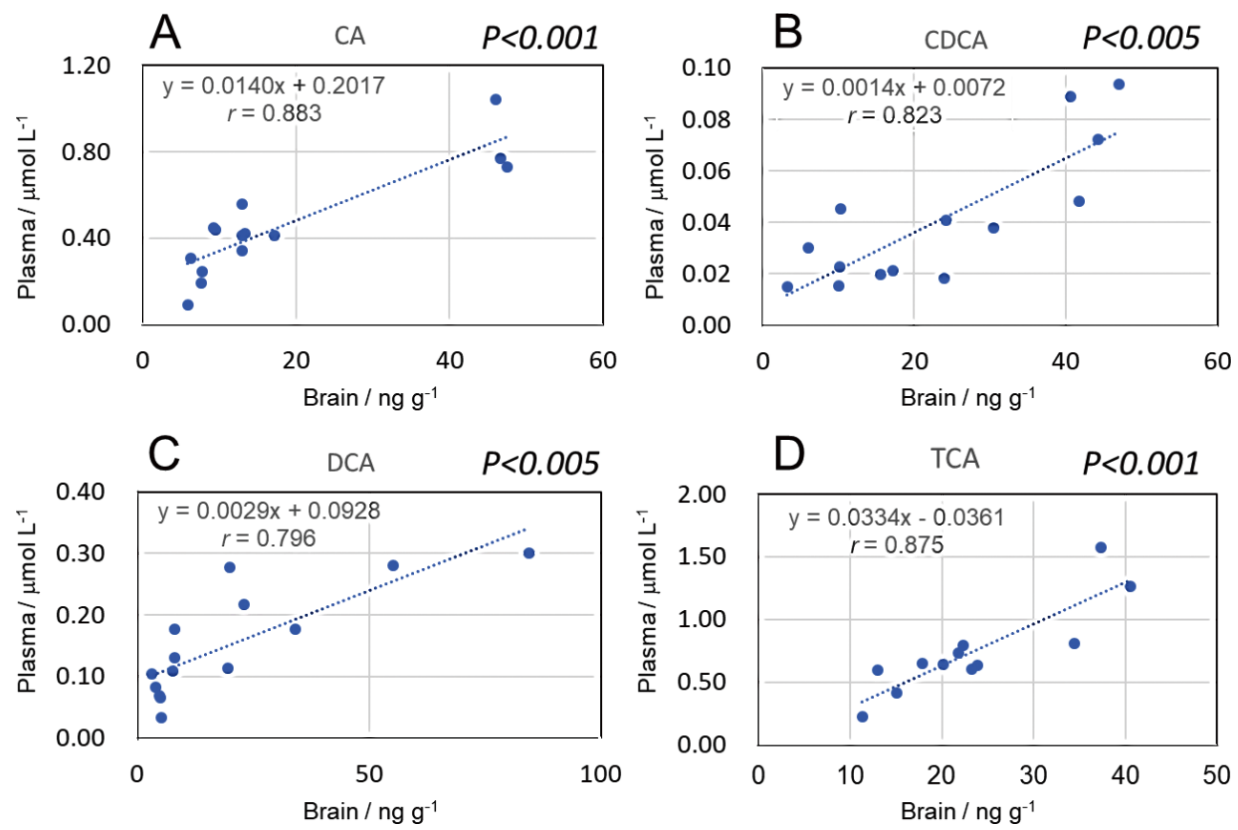

Fig. 4 Correlations between the four bile acid concentrations in the mouse brain and plasma. A: CA, B: CDCA, C: DCA, and D: TCA. $p$ value $<0.05$ was considered statistically significant.

the present method (Fig. 2a). As shown in Fig. 3a, three unconjugated BAs (CA, CDCA, and DCA) and a taurine conjugated BA (TCA) could be quantified in the brain of an 8 -week-old male mice. Although there are large individual differences in the concentrations of the four BAs, the average concentrations of CA, CDCA, and DCA in the mouse brains are close to the concentrations previously reported in rat brains. ${ }^{9}$ As a new finding, it was revealed that TCA exists in normal young mouse brains (Figs. 2a and 3a). In contrast, all the targeted BAs, including LCA, were detected above the LOQ (Fig. 2b), and quantified in the plasma (Fig. 3b). As shown in Fig. 2b, UDCA (isomer of CDCA and DCA), $\beta$-MCA (isomer of CA), and T- $\beta$-MCA (isomer of TCA) were observed on the chromatograms obtained from mouse plasma.

Furthermore, we examined the correlation of each BA concentration between the mouse brain and plasma. As shown in Fig. 4, significant positive correlations were observed between the brain and plasma concentrations of CA, DCA, CDCA, and TCA. These data indicate that each BA concentration in the plasma reflects the corresponding BA in the mouse brain. Higashi et al. reported good correlations between the rat brain and serum concentrations of CA, CDCA and DCA. ${ }^{9}$ They also suggested that most of the BAs in the brain are derived from the periphery. Thus, when the plasma concentration of the specific BA that is expected to be a biomarker of $\mathrm{AD}$ fluctuates, the corresponding BA also seems to be varied similarly in the brain. Although it is still uncertain whether there are positive correlations between the human brain and plasma concentrations of BAs, the significance of individual analysis of bile acid appears clear for future studies for elucidating their homeostatic mechanisms and participation in various brain and nerve system disorders.

\section{Conclusions}

In this study, we developed a suitable method using LC-MS/MS analysis to determine the five bile acids that are associated with
AD. The established method of simultaneous analysis of the five BAs has good accuracy, sensitivity, and linearity ranges. Results showed that among the five BAs, DCA, CA, TCA, and DCDA were quantitatively determined in the mouse brain and plasma. These analytical data demonstrated that there were positive correlations between the plasma and brain concentrations of the four BAs in mice. These results suggest that each BA plasma level reflects its level in the brain. Therefore, measuring their concentrations in the plasma may be helpful for the investigation of the pathological state in $\mathrm{AD}$ patients and mouse model of $\mathrm{AD}$.

\section{Conflicts of Interest}

The authors declare no conflict of interest.

\section{Supporting Information}

This material is available free of charge on the Web at http:// www.jsac.or.jp/analsci/.

\section{Acknowledgements}

This work was supported in part by JSPS KAKENHI, grant numbers JP19K07208 and JP19K1642.

\section{References}

1. S. M. Houten, M. Watanabe, and J. Auwerx, EMBO J., 2006, 25, 1419.

2. T. Q. de Aguiar Vallim, E. J. Tarling, and P. A. Edwards, Cell Metab., 2013, 17, 657.

3. F. Kuipers, V. W. Bloks, and A. K. Groen, Nat. Rev. Endocrinol., 2014, 10, 488. 
4. K. L. Mertens, A. Kalsbeek, M. R. Soeters, and H. M Eggink, Front. Neurosci., 2017, 11, 617.

5. W. A. Alrefai and R. K. Gill, Pharm. Res., 2007, 24, 1803.

6. D. Nizamutdinov, S. DeMorrow, M. McMillin, J. Kain, S. Mukherjee, S. Zeitouni, G. Frampton, P. C. Bricker, J. Hurst, and L. A. Shapiro, Sci. Rep., 2017, 7, 40112.

7. X. Zheng, T. Chen, A. Zhao, X. Wang, G. Xie, F. Huang, J. Liu, Q. Zhao, S. Wang, and C. Wang, Sci. Rep., 2016, 6 , 24125.

8. N. Mano, T. Goto, M. Uchida, K. Nishimura, M. Ando, N. Kobayashi, and J. Goto, J. Lipid Res., 2004, 45, 295.

9. T. Higashi, S. Watanabe, K. Tomaru, W. Yamazaki, K. Yoshizawa, S. Ogawa, H. Nagao, K. Minato, M. Maekawa, and N. Mano, Steroids, 2017, 125, 107.

10. S. Ferdinandusse, S. Denis, P. L. Faust, and R. J. Wanders, J. Lipid Res., 2009, 50, 2139.

11. X. Pan, C. T. Elliott, B. McGuinness, P. Passmore, P. G. Kehoe, C. Hölscher, P. L. McClean, S. F. Graham, and B D. Green, Metabolites, 2017, 7, pii: E28.

12. S. Mahmoudian Dehkordi, M. Arnold, K. Nho, S. Ahmad, W. Jia, G. Xie, G. Louie, A. Kueider-Paisley, M. A. Moseley, J. W. Thompson, L. St John Williams, J. D. Tenenbaum, C. Blach, R. Baillie, X. Han, S. Bhattacharyya, J. B. Toledo, S. Schafferer, S. Klein, T. Koal, S. L. Risacher, M. A. Kling, A. Motsinger-Reif, D. M. Rotroff, J. Jack, T.
Hankemeier, D. A. Bennett, P. L. De Jager, J. Q. Trojanowski, L. M. Shaw, M. W. Weiner, P. M. Doraiswamy, C. M. van Duijn, A. J. Saykin, G. Kastenmüller, and R. Kaddurah-Daouk, Alzheimers Dement., 2019, 15, 76.

13. J. Olazarán, L. Gil-de-Gómez, A. Rodríguez-Martín, M Valentí-Soler, B. Frades-Payo, J. Marín-Muñoz, C. Antúnez, A. Frank-García, C. Acedo-Jiménez, L. Morlán-Gracia, R. Petidier-Torregrossa, M. C. Guisasola, F. Bermejo-Pareja, Á. Sánchez-Ferro, D. A. Pérez-Martínez, S. ManzanoPalomo, R. Farquhar, A. Rábano, and M. Calero, J. Alzheimers Dis., 2015, 45, 1157.

14. J. Marksteiner, I. Blasko, G. Kemmler, T. Koal, and C. Humpel, Metabolomics, 2018, 14, 1.

15. F. H. Bazzari, D. M. Abdallah, and H. S. El-Abhar, Molecules, 2019, 24, 1992.

16. Y. Alnouti, I. L. Csanaky, and C. D. Klaassen, J. Chromatogr. $B$, 2008, 873, 209

17. R. Thakare, J. A. Alamoudi, N, Gautam, A. D. Rodrigues, and Y. Alnouti, J. Appl. Toxicol., 2018, 38, 1336.

18. J. Han, Y. Liu, R. Wang, J. Yang, V. Ling, and C. H. Borchers, Anal. Chem., 2015, 87, 1127.

19. L. Luo, J. Aubrecht, D. Li, R. L. Warner, K. J. Johnson, J. Kenny, and J. L. Colangelo, PLoS One, 2018, 13, e0193824.

20. A. Jo, S. Koyagi, W. Hobo, S. Otani, S. Ogawa, and T. Higashi, Anal Sci., 2020, 36, 1099. 